Bull. Austral. Math. Soc.

VOL. 50 (1994) [225-243]

\title{
AN APPLICATION OF JACOBI TYPE POLYNOMIALS TO IRRATIONALITY MEASURES
}

\author{
Ari Heimonen, Tapani Matala-Aho and Keijo Vä̈̈nÄnen
}

The paper provides irrationality measures for certain values of binomial functions and definite integrals of some rational functions. The results are obtained using Jacobi type polynomials and divisibility considerations of their coefficients.

\section{Introduction}

In the previous work [13] we considered the irrationality measures of the values of the Gauss hypergeometric series

$$
F(z)={ }_{2} F_{1}\left(\begin{array}{c|}
1, b \\
c
\end{array} \mid z\right)=\sum_{n=0}^{\infty} \frac{(b)_{n}}{(c)_{n}} z^{n}
$$

where $b, c \neq 0,-1,-2, \ldots$ are rational parameters, and $(b)_{0}=1,(b)_{n}=b(b+1)$ $\ldots(b+n-1), n=1,2, \ldots$. The general result of the work [13] was sharpened in the case of the logarithmic function by using the divisibility properties of the coefficients of the approximation polynomials. This idea was first realised for the binomial function by Chudnovsky [5], and then for the logarithmic function by Rukhadze [15], see also Dubitskas [7] and Hata [10]. In this paper we apply the results of [13], especially the general divisibility criterion, to the case $b=1 / k, c=1+1 / k(k \geqslant 2)$ and to the binomial case $b=1 / k, c=1(k \geqslant 3)$ in order to get sharper irrationality measures both in the archimedean and $p$-adic case. For example in the first case we achieve generalisations of the theorems of Chudnovsky [4], Danilov [6], Dubitskas [7], Hata $[10,11]$ and Huttner [14]. In the binomial case we obtain the archimedean results of Chudnovsky [5] (see also Easton [8]), which considerably improve the work of Baker [2], and p-adic analogues improving the work of Bundschuh [3].

Received 10th November, 1993

Copyright Clearance Centre, Inc. Serial-fee code: $0004-9729 / 94 \$ \$ A 2.00+0.00$. 


\section{NOTATION AND RESUltS}

We shall denote by $\mathbb{Q}_{v}$ the $v$-adic completion of $\mathbb{Q}$, where $v \in\{\infty$, primes $p\}$, in particular $\mathbb{Q}_{\infty}=\mathbb{R}$. For an irrational number $\theta \in \mathbb{Q}_{v}$, by an irrationality measure $m_{v}(\theta)$ of $\theta$ we mean the infimum of all $m$ satisfying the following condition: for any $\varepsilon>0$ there exists an $H_{0}=H_{0}(\varepsilon)$ such that

$$
\left|\theta-\frac{P}{Q}\right|_{v}>H^{-m-\varepsilon}
$$

for all rationals $P / Q$ satisfying $H=\max \{|P|,|Q|\}>B_{0}$. In the following we denote $m_{\infty}(\theta)=m(\theta)$. All our measures are effective in the sense that $H_{0}$ can be effectively determined.

In our first case we have $b=1 / k, c=1+1 / k$, where $k \geqslant 2$ is a natural number. For a given rational $r / s,(r, s)=1$, we denote the denominators of $(s-r) / k$ and $(s-r) /\left(k \prod_{p \mid k} p\right)$ by $k^{*}$ and $k^{* *}$, respectively. Further, with a given rational $\beta \geqslant 1$ we define

$$
\begin{aligned}
A(\beta, z) & =\min _{0<\rho<|z|+\frac{1}{2}(1-\operatorname{sgn} z)}\left(\frac{(\rho+|z|)(\rho+|z|-\operatorname{sgn} z)^{\beta}}{\rho}\right) \quad(z<0 \text { or } z \geqslant 1) \\
S(\beta, z) & =\max _{0 \leqslant t \leqslant 1} \frac{t(1-t)^{\beta}}{1-z t} \quad(-1 \leqslant z<1) \\
\omega_{1}(\beta) & =k \mu_{k} \min \left\{\left(\frac{k^{*}}{k}\right)^{\beta},\left(\frac{k^{* *}}{k \mu_{k}}\right)^{\beta}\right\} e^{\beta \lambda(k)-\sigma(\beta, k)}, \\
Q(\beta, k) & =\omega_{1}(\beta)|r|^{\beta} A\left(\beta, \frac{s}{r}\right), \quad R(\beta, k)=\omega_{1}(\beta)|r|^{1+\beta} s^{-1} S\left(\beta, \frac{r}{s}\right)
\end{aligned}
$$

where

$$
\mu_{h}=\prod_{p \mid h} p^{1 /(p-1)}, \quad \lambda(h)=\frac{h}{\phi(h)} \sum_{\substack{i=1 \\(i, h)=1}}^{h} \frac{1}{i} \quad\left(h \in \mathbb{Z}^{+}\right)
$$

and $\sigma(\beta, k)$ is given in the formula (19). Then the following theorem holds.

THEOREM 1. Let $r / s \in(-1,1)$ be a rational number satisfying $(r, s)=1$. Then

$$
m\left({ }_{2} F_{1}\left(\begin{array}{c}
1, \frac{1}{k} \\
1+\frac{1}{k}
\end{array} \frac{r}{s}\right)\right) \leqslant \inf _{\beta}^{*}\left\{1-\frac{\ln Q(\beta, k)}{\ln R(\beta, k)}\right\},
$$

where $\inf _{\beta}^{*}$ means that for a given $r / s$ the infimum is taken over all rationals $\beta \geqslant 1$ satisfying $R(\beta, k)<1$.

We note that the choice $\beta=1$ implies

$$
m\left({ }_{2} F_{1}\left(\begin{array}{c|c}
1, \frac{1}{k} & \left.\frac{r}{s}\right) \\
1+\frac{1}{k} & \frac{s}{s}
\end{array}\right) \leqslant 1-\frac{2 \ln (\sqrt{s}+\sqrt{s-r})+\lambda(k)+\ln \min \left\{k^{*} \mu_{k}, k^{* *}\right\}}{2 \ln |\sqrt{s}-\sqrt{s-r}|+\lambda(k)+\ln \min \left\{k^{*} \mu_{k}, k^{* *}\right\}}\right.
$$


for all rationals $r / s \in(-1,1)$ satisfying

$$
\min \left\{k^{*} \mu_{k}, k^{* *}\right\} e^{\lambda(k)}(\sqrt{s}-\sqrt{s-r})^{2}<1
$$

This is a sharpening of Huttner's Theorem [14]. In Hata [11] some improvements of Huttner's results were obtained under the assumption $r=1 \equiv s\left(\bmod k \prod_{p \mid k} p\right)$, which implies $k^{* *}=1$. Further, we note that the numbers in Theorem 1 are definite integrals of the form

$$
\frac{1}{x} \int_{0}^{x} \frac{d t}{1 \pm t^{k}}
$$

and the transcendence of this kind of numbers was considered by van der Poorten [16] using Baker's results on linear forms of logarithms.

Some numerical examples are given in the table of the following page.

Some of the numbers given explicitly in the table have been considered in Hata $[10,11]$, where the bounds are the same as we have (compere also with Huttner [14]). Recently Hata has achieved an improvement $m(\pi / \sqrt{3}) \leqslant 4.601579 \ldots$ in the work [12], where he also reached a remarkable result $m(\pi) \leqslant 8.0161$. Further, we note that the table contains some examples where the value $\beta=1$ does not give any measure.

Our $p$-adic result is

TheOREM $1 p$. Let $p \nmid k$ be a prime and let us assume $|r / s|_{p}<1$.

(1) If $r / s>1$ is a rational number satisfying

$$
\min \left\{k^{*} \mu_{k}, k^{* *}\right\} e^{\lambda(k)} r|r|_{p}^{2}<1
$$

then

$$
m_{p}\left({ }_{2} F_{1}\left(\begin{array}{c|c}
1, \frac{1}{k} & r \\
1+\frac{1}{k} & \frac{r}{s}
\end{array}\right)\right) \leqslant \frac{2 \ln |r|_{p}}{2 \ln |r|_{p}+\ln r+\lambda(k)+\ln \min \left\{k^{*} \mu_{k}, k^{* *}\right\}} .
$$

(2) If $r / s<1$ is a rational number satisfying

$$
\min \left\{k^{*} \mu_{k}, k^{* *}\right\} e^{\lambda(k)}(\sqrt{s}+\sqrt{s-r})^{2}|r|_{p}^{2}<1,
$$

then

$$
m_{p}\left({ }_{2} F_{1}\left(\begin{array}{c}
1, \frac{1}{k} \\
1+\frac{1}{k}
\end{array} \mid \frac{r}{s}\right)\right) \leqslant \frac{2 \ln |r|_{p}}{2 \ln |r|_{p}+2 \ln |\sqrt{s}+\sqrt{s-r}|+\lambda(k)+\ln \min \left\{k^{*} \mu_{k}, k^{* *}\right\}}
$$




\begin{tabular}{|c|c|c|c|c|c|}
\hline$\frac{r}{8}$ & $k$ & number & $\beta$ & upper bound & $\beta=1$ \\
\hline$-\frac{1}{3}$ & 2 & $\frac{\pi}{\sqrt{3}}$ & $\frac{3}{2}$ & $5.0874 \ldots$ & $8.3099 \ldots$ \\
\hline$\frac{3}{13}$ & 2 & & $\frac{6}{5}$ & $97.719 \ldots$ & - \\
\hline$-\frac{1}{7}$ & 2 & $\sqrt{7} \arctan \frac{1}{\sqrt{7}}$ & $\frac{4}{3}$ & $4.0298 \ldots$ & $4.8569 \ldots$ \\
\hline$\frac{1}{45}$ & 2 & $\sqrt{5} \log \frac{3 \sqrt{5}+1}{3 \sqrt{5}-1}$ & $\frac{9}{8}$ & $3.1346 \ldots$ & $3.2571 \ldots$ \\
\hline$\frac{1}{8}$ & 2 & $\sqrt{2} \log \frac{2 \sqrt{2}+1}{2 \sqrt{2}-1}$ & $\frac{7}{6}$ & $41.032 \ldots$ & $493.12 \ldots$ \\
\hline$\frac{1}{5}$ & 2 & $\sqrt{5} \log \frac{\sqrt{5}+1}{\sqrt{5}-1}$ & $\frac{3}{2}$ & $4.4937 \ldots$ & $6.5082 \ldots$ \\
\hline$-\frac{2}{7}$ & 3 & & $\frac{7}{6}$ & $84.960 \ldots$ & - \\
\hline$-\frac{1}{5}$ & 3 & & $\frac{4}{3}$ & $11.531 \ldots$ & $21.327 \ldots$ \\
\hline$-\frac{1}{8}$ & 3 & & $\frac{4}{3}$ & $4.5586 \ldots$ & $5.5280 \ldots$ \\
\hline$\frac{2}{25}$ & 3 & & $\frac{7}{6}$ & $6.7886 \ldots$ & $7.8411 \ldots$ \\
\hline$\frac{1}{7}$ & 3 & & $\frac{4}{3}$ & $9.2848 \ldots$ & $14.242 \ldots$ \\
\hline$\frac{1}{4}$ & 3 & & $\frac{4}{3}$ & $30.435 \ldots$ & - \\
\hline$-\frac{1}{7}$ & 4 & & $\frac{4}{3}$ & $6.6372 \ldots$ & $9.2718 \ldots$ \\
\hline$\frac{3}{29}$ & 4 & & $\frac{10}{9}$ & $94.453 \ldots$ & - \\
\hline$-\frac{1}{11}$ & 4 & & $\frac{8}{7}$ & $12.086 \ldots$ & $16.347 \ldots$ \\
\hline$\frac{1}{64}$ & 4 & $\log \frac{13}{5}+2 \arctan \frac{4}{7}$ & $\frac{28}{27}$ & $13.164 \ldots$ & $13.764 \ldots$ \\
\hline$\frac{1}{81}$ & 4 & $\log 2+\arctan \frac{3}{4}$ & $\frac{12}{11}$ & $3.6073 \ldots$ & $3.7160 \ldots$ \\
\hline$\frac{1}{9}$ & 4 & $\sqrt{3}\left(\log (2+\sqrt{3})+\frac{\pi}{3}\right)$ & $\frac{4}{3}$ & $6.1382 \ldots$ & $8.2100 \ldots$ \\
\hline$-\frac{1}{4}$ & 5 & & $\frac{5}{4}$ & $33.251 \ldots$ & - \\
\hline$-\frac{1}{9}$ & 5 & & $\frac{5}{4}$ & $8.5809 \ldots$ & $11.538 \ldots$ \\
\hline$\frac{1}{24}$ & 5 & & $\frac{6}{5}$ & $4.2604 \ldots$ & $4.6295 \ldots$ \\
\hline$\frac{1}{31}$ & 5 & & $4.9971 \ldots$ & $5.3453 \ldots$ \\
\hline$\frac{1}{11}$ & 5 & & $\frac{5}{9}$ & $7.8909 \ldots$ & $10.230 \ldots$ \\
\hline$\frac{1}{35}$ & 6 & & $6.5200 \ldots$ & $7.3106 \ldots$ \\
\hline$\frac{1}{49}$ & 6 & & $16.211 \ldots$ & $18.508 \ldots$ \\
\hline
\end{tabular}


Our second case considers the binomial function. Let $b=1 / k, c=1$, where $k \geqslant 3$ is again a natural number, and for a given $r \in \mathbb{Z}$ let us denote the denominators of $r / k$ and $r /\left(k \prod_{p \mid k} p\right)$ by $k_{*}$ and $k_{* *}$, respectively. Further, let

$$
\tau(k)=\frac{2}{\phi(k)} \sum_{\substack{k / 2<q<k \\(q, k)=1}}\left(\Psi(1)-\Psi\left(\frac{q}{k}\right)\right),
$$

where $\Psi$ is the digamma function, see [9].

Theorem 2. If $r / s \in(-1,1)$ satisfies

$$
\min \left\{k_{*} \mu_{k}, k_{* *}\right\} e^{-\tau(k)}(\sqrt{s}-\sqrt{s-r})^{2}<1,
$$

then

$$
m\left(\left(1-\frac{r}{s}\right)^{-1 / k}\right) \leqslant 1-\frac{2 \ln (\sqrt{s}+\sqrt{s-r})-\tau(k)+\ln \min \left\{k_{*} \mu_{k}, k_{* *}\right\}}{2 \ln |\sqrt{s}-\sqrt{s-r}|-\tau(k)+\ln \min \left\{k_{*} \mu_{k}, k_{* *}\right\}} .
$$

For example we have $m(\sqrt[3]{2}) \leqslant \underline{2.4297 \ldots}$ and $m(\sqrt[3]{6}) \leqslant 2.3205 \ldots$, which are among the examples given by Chudnovsky [5, p.377]. In addition we obtain $m(\sqrt[4]{5}) \leqslant$ 2.7473 $\ldots, \quad r / s=1 / 81, \quad m(\sqrt[7]{17}) \leqslant 5.0311 \ldots, r / s=11 / 3^{7}$, and $m(\sqrt[7]{127}) \leqslant$ 2.70468 .,$r / s=1 / 2^{7}$, where the underlined bounds are better than Thue's bound.

In the $p$-adic case we have an improvement of the work of Bundschuh [3].

ThEOREM $2 p$. Let $p \nmid k$ be a prime and let us assume $|r / s|_{p}<1$.

(1) If $r / s>1$ and

$$
\min \left\{k_{*} \mu_{k}, k_{* *}\right\} e^{-\tau(k)} r|r|_{p}^{2}<1
$$

then

$$
m_{p}\left(\left(1-\frac{r}{s}\right)^{-1 / k}\right) \leqslant \frac{2 \ln |r|_{p}}{2 \ln |r|_{p}+\ln r-\tau(k)+\ln \min \left\{k_{*} \mu_{k}, k_{* *}\right\}} .
$$

Especially we have

$$
m_{p}\left(\left(1-p^{l}\right)^{-1 / k}\right) \leqslant \frac{2 l \ln p}{l \ln p+\tau(k)-\ln k \mu_{k}}
$$

for all $p^{l}>k \mu_{k} e^{-\tau(k)}$.

(2) If $r / s<1$ and

$$
\min \left\{k_{*} \mu_{k}, k_{* *}\right\} e^{-\tau(k)}(\sqrt{s}+\sqrt{s-r})^{2}|r|_{p}^{2}<1
$$

then

$$
m_{p}\left(\left(1-\frac{r}{s}\right)^{-1 / k}\right) \leqslant \frac{2 \ln |r|_{p}}{2 \ln |r|_{p}+2 \ln |\sqrt{s}+\sqrt{s-r}|-\tau(k)+\ln \min \left\{k_{*} \mu_{k}, k_{* *}\right\}} .
$$


For example

$$
\begin{array}{ll}
m_{2}(\sqrt[3]{3}) \leqslant 2.4597 \ldots, \frac{r}{s}=\frac{128}{125}, \quad m_{2}(\sqrt[3]{5}) \leqslant 2.7088 \ldots, \frac{r}{s}=\frac{32}{27} \\
m_{2}(\sqrt[3]{41}) \leqslant 2.4597 \ldots, \frac{r}{s}=\frac{3 \cdot 128}{343}, \quad m_{5}(\sqrt[3]{11}) \leqslant 2.7845 \ldots, \frac{r}{s}=\frac{3 \cdot 25}{64} \\
m_{3}(\sqrt[4]{-23}) \leqslant 3.1125 \ldots, \frac{r}{s}=\frac{8 \cdot 3^{4}}{5^{4}}, \quad m_{5}(\sqrt[4]{6}) \leqslant 3.2746 \ldots, \frac{r}{s}=\frac{2 \cdot 5^{3}}{4^{4}}<1 \\
m_{3}(\sqrt[4]{13}) \leqslant 3.1717 \ldots, \frac{r}{s}=\frac{3^{5}}{2^{8}}<1, & m_{5}(\sqrt[4]{46}) \leqslant 2.8704 \ldots, \frac{r}{s}=\frac{2 \cdot 5^{4}}{6^{4}}<1, \\
m_{2}(\sqrt[5]{13}) \leqslant 2.6349 \ldots, \frac{r}{s}=\frac{2^{8}}{3^{5}}, & m_{2}(\sqrt[5]{53}) \leqslant 3.2736 \ldots, \frac{r}{s}=\frac{3 \cdot 4^{5}}{5^{5}}<1 .
\end{array}
$$

\section{PADÉ TYPE APPROXIMATIONS}

Throughout this paper we shall assume that $c>b>0, b=a / f, c=g / h$, where $a, f, g, h$ are natural numbers such that $(a, f)=(g, h)=1$. Let us denote $B=b-1=E / F, C=c-b-1=G / H$ with $E, G \in \mathbb{Z}, F, H \in \mathbb{N},(E, F)=(G, H)=1$. Further, let $l, m$ and $n$ denote positive integer parameters satisfying $l \leqslant \min \{m, n\}$.

By using the Jacobi type polynomial

$$
A_{l, m, n}(z)=\frac{1}{z^{B}(1-z)^{C}} \frac{1}{l !}\left(\frac{d}{d z}\right)^{l}\left(z^{n+B}(1-z)^{m+C}\right)
$$

and the integral representation

$$
F(z)=\int_{0}^{1} \frac{\omega(t)}{1-z t} d t, \quad \omega(t)=\frac{\Gamma(c)}{\Gamma(c-b) \Gamma(b)} t^{B}(1-t)^{C} \quad(|z|<1)
$$

we obtained in [13] the approximation formula

$$
R_{l, m, n}(z)=Q_{l, m, n}(z) F(z)-P_{l, m, n}(z)
$$

where

$$
\begin{aligned}
Q_{l, m, n}(z) & =z^{n+m-l} A_{l, m, n}\left(\frac{1}{z}\right)=(-1)^{l}(z-1)^{m-l} \sum_{k=0}^{l}\left(\begin{array}{c}
n+B \\
k
\end{array}\right)\left(\begin{array}{c}
m+C \\
l-k
\end{array}\right)(1-z)^{k}, \\
P_{l, m, n}(z) & =z^{n+m-l-1} \int_{0}^{1} \frac{A_{l, m, n}(1 / z)-A_{l, m, n}(t)}{1 / z-t} \omega(t) d t \\
R_{l, m, n}(z) & =z^{n+m-l} \int_{0}^{1} \frac{A_{l, m, n}(t) \omega(t)}{1-z t} d t \\
& =(-1)^{l} z^{n+m} \frac{(b)_{n}(c-b)_{m}}{(c)_{n+m}} 2 F_{1}\left(\begin{array}{c}
l+1, n+b \\
n+m+c
\end{array} \mid z\right) .
\end{aligned}
$$


In the following we suppose that $l=n, m=[\beta n]$, where $\beta \geqslant 1$ is a rational number, and we use the notation

$$
P_{n}(z)=P_{l, m, n}(z), \quad Q_{n}(z)=Q_{l, m, n}(z), \quad R_{n}(z)=R_{l, m, n}(z) .
$$

Further, let $\delta=\delta(v)$ be 1 , if $v=\infty$, and 0 , if $v$ is finite. By $c_{1}, c_{2}, \ldots$ we shall denote positive constants independent of $n$. We now have the following lemma.

LEMMA 1.

(i) If $|z|_{v}<1$ and in the finite case $v \not f h$, then we have

$$
\left|R_{n}(z)\right|_{v} \leqslant c_{1} n^{1-\delta}\left(|z|_{v}^{1+\beta} S(\beta, z)^{\delta}\right)^{n}
$$

for all $n \geqslant c_{2}$. In the archimedean case the bound on the right-hand side of this inequality is an asymptotic for $\left|R_{n}(z)\right|(n \rightarrow \infty)$, and the bound holds at $z=-1$, too.

(ii) If $z \in[-1,1)$ and $S(\beta, z) \leqslant A(\beta, 1 / z) /|z|$, then

$$
\max \left\{\left|Q_{n}(z)\right|,\left|P_{n}(z)\right|\right\} \leqslant c_{3}\left(|z|^{\beta} A\left(\beta, \frac{1}{z}\right)\right)^{n} .
$$

(iii) If $q=\max \{B, C\} \geqslant-1 / 2, \beta=1$ and $z>1$, then

$$
\max \left\{\left|Q_{n}(z)\right|,\left|P_{n}(z)\right|\right\} \leqslant c_{4} n^{q+2}|z|^{n} .
$$

(iv) If $\beta=1$ and $z<-1$, then

$$
\max \left\{\left|Q_{n}(z)\right|,\left|P_{n}(z)\right|\right\} \leqslant c_{5}\left(|z| A\left(1, \frac{1}{z}\right)\right)^{n} .
$$

Further, all these bounds (i)-(iv) with the value $\beta=1$ are true, if $P_{n}(z), Q_{n}(z)$ and $R_{n}(z)$ are replaced by

$$
\widehat{P}_{n}(z)=P_{n, n, n+1}(z), \quad \widehat{Q}_{n}(z)=Q_{n, n, n+1}(z), \quad \widehat{R}_{n}(z)=R_{n, n, n+1}(z),
$$

respectively.

Proof: Our lemma follows immediately from Lemmas 1 and 3 of [13]. We only need to note that the the polynomial $A_{n, n, n+1}(z)$ with parameters $B$ and $C$ equals the polynomial $z A_{n, n, n}(z)$ with parameters $1+B$ and $C$.

LEMMA 2. If $\beta=1$, then

$$
\left|\begin{array}{cc}
Q_{n}(z) & P_{n}(z) \\
Q_{n+1}(z) & P_{n+1}(z)
\end{array}\right|=(-1)^{n} \frac{(b)_{n}(c-b)_{n}}{(c)_{2 n}}\left(\begin{array}{c}
-n-c \\
n+1
\end{array}\right) z^{2 n}
$$

and

$$
\left|\begin{array}{cc}
\widehat{Q}_{n}(z) & \widehat{P}_{n}(z) \\
\widehat{Q}_{n+1}(z) & \widehat{P}_{n+1}(z)
\end{array}\right|=(-1)^{n} \frac{(b)_{n+1}(c-b)_{n}}{(c)_{2 n+1}}\left(\begin{array}{c}
-n-c-1 \\
n+1
\end{array}\right) z^{2 n+1}
$$

The first equality is proved in [13, Lemma 6], and the other can be proved similarly. 
4. ON The ARITHMEtic PROPERTIES OF ThE COEFficients of $P_{n}$ AND $Q_{n}$ From the definition of $P_{n}$ and $Q_{n}$ it follows that (see [13])

$$
\begin{aligned}
& Q_{n}(z)=\sum_{j=0}^{n}(-1)^{m} a_{j}(1-z)^{m-n+j}, \\
& P_{n}(z)=-z^{m-1} \sum_{j=m-n}^{m} c_{j} \sum_{i=0}^{j-1}\left(\frac{z-1}{z}\right)^{j-1-i} \frac{(C+1) \ldots(C+i)}{c(c+1) \ldots(c+i-1)},
\end{aligned}
$$

where

$$
a_{j}=\left(\begin{array}{c}
n+B \\
j
\end{array}\right)\left(\begin{array}{c}
m+C \\
n-j
\end{array}\right), \quad c_{j}=\sum_{i=0}^{j-m+n}(-1)^{m-j} a_{n-m+j-i}\left(\begin{array}{c}
m-j+i \\
i
\end{array}\right)
$$

Let us consider our first case, when $B=1 / k-1, C=0$. As in the proof of Lemma 4 in [13] we have

$$
\begin{gathered}
a_{j} \in \frac{1}{k^{j} \mu_{k}(j)} \mathbb{Z} \quad(j=0, \ldots, n), \\
c_{j} \in \frac{1}{k^{j-m+n} \mu_{k}(j-m+n)} \mathbb{Z} \quad(j=m-n, \ldots, m), \\
\frac{k^{i} i !}{(1+k) \ldots(1+i k)} \in \frac{k^{i} \mu_{k}(i)}{d_{i}(k)} \mathbb{Z},
\end{gathered}
$$

where we have used the notation

$$
\mu_{k}(i)=\prod_{p \mid k} p^{v_{p}(i !)}, \quad d_{i}(k)=\operatorname{lcm}\{1+k, \ldots, 1+i k\}
$$

(As usual, if $r \in \mathbb{Q}, r \neq 0$, then $v_{p}(r)$ is defined by $r=p^{v_{p}(r)} R / S$, where $(R, S)=$ $(R, p)=(S, p)=1$.) Thus the terms in the sum presenting $P_{n}(r / s)$ are of the form

$$
c_{j}\left(\frac{r-s}{r}\right)^{j-i-1} \frac{k^{i} i !}{(1+k) \ldots(1+i k)} \in \frac{(r-s)^{j-i} r k^{m-n} \mu_{k}(i)}{(r-s)(r k)^{j-i} \mu_{k}(j-m+n) d_{i}(k)} \mathbb{Z},
$$

where we used (8) to obtain the last expression. Since

$$
\begin{aligned}
v_{p}((m-n) !)+ & v_{p}((j-m+n) !)-v_{p}(i !)-v_{p}((j-i) !) \\
\leqslant & \sum_{k \leqslant\left[\frac{\ln m}{\ln p}\right]}\left(\left[\frac{m-n}{p^{k}}\right]+\left[\frac{j-m+n}{p^{k}}\right]-\left[\frac{i}{p^{k}}\right]-\left[\frac{j-i}{p^{k}}\right]\right) \leqslant 2\left[\frac{\ln m}{\ln p}\right],
\end{aligned}
$$


it follows that the above expression belongs to

$$
\frac{k^{m-n} \mu_{k}(m-n)}{(r-s) r^{j-1-i} d_{i}(k) \mu_{k}(j-i) \prod_{p \mid k} p^{C(n)}}\left(\frac{r-s}{k}\right)^{j-i} \mathbb{Z}
$$

where $C(n)=2\left[\frac{\ln \beta n}{\ln p}\right]$. Further, by (6), the terms in the sum giving $Q_{n}(r / s)$ satisfy

$$
a_{j}\left(\frac{r-s}{s}\right)^{m-n+j} \in \frac{k^{m-n} \mu_{k}(m-n)(r-s)^{m-n+j}}{s^{m-n+j} k^{m-n+j} \mu_{k}(m-n+j)} \mathbb{Z}
$$

These considerations imply

Lemma 3. Suppose that $B=1 / k-1, C=0$. If

$$
\Omega_{n, 1}=\frac{(r-s) s^{m} d_{m}(k) \prod_{p \mid k} p^{C(n)}}{k^{m-n} \mu_{k}(m-n)} \min \left\{\left(k^{*}\right)^{m} \mu_{k}(m),\left(k^{* *}\right)^{m}\right\}
$$

then

$$
\Omega_{n, 1} Q_{n}\left(\frac{r}{s}\right), \quad \Omega_{n, 1} P_{n}\left(\frac{T}{s}\right) \in \mathbb{Z}
$$

Next we consider our second case, where $B=(1-k) / k, C=-1 / k$. By the Gauss summation formula (see $[9$, p.104])

$$
{ }_{2} F_{1}\left(\begin{array}{c|c}
a,-n & 1 \\
c & 1
\end{array}\right)=\frac{(c-a)_{n}}{(c)_{n}} \quad(c \neq 0,-1, \ldots)
$$

it follows that

$$
\widehat{Q}_{n}(z)=\sum_{j=0}^{n}\left(\begin{array}{c}
n+\frac{1}{k} \\
j
\end{array}\right)\left(\begin{array}{c}
n-\frac{1}{k} \\
n-j
\end{array}\right)(1-z)^{j}=\sum_{j=0}^{n}\left(\begin{array}{c}
n+\frac{1}{k} \\
j
\end{array}\right)\left(\begin{array}{c}
2 n-j \\
n-j
\end{array}\right)(-z)^{j} .
$$

Thus

$$
\widehat{Q}_{n}\left(\frac{r}{s}\right) \in \frac{1}{s^{n} k^{n} \mu_{k}(n)} \mathbb{Z}
$$

This result can be improved in many cases. Namely, $\widehat{Q}_{n}(r / s)$ is the sum of the terms

$$
\left(\begin{array}{c}
2 n-j \\
n-j
\end{array}\right) \frac{(1+(n-j+1) k) \ldots(1+n k)}{j !}\left(\frac{-r}{k s}\right)^{j}
$$

belonging to

$$
\frac{1}{s^{j} \mu_{k}(j)}\left(\frac{r}{k}\right)^{j} \mathbb{Z}
$$


Since $F(z)=(1-z)^{-1 / k}$ it follows that $\widehat{P}_{n}(z)=\lambda Q_{n}^{*}(z)$ where $\lambda$ is a constant and $Q_{n}^{*}$ is obtained from $\widehat{Q}_{n}$ by replacing $1 / k$ by $-1 / k$. Further

$$
\left[\widehat{Q}_{n}(z) F(z)\right]_{n}=\widehat{P}_{n}(z)
$$

(if $f(z)=\sum_{j=0}^{\infty} f_{j} z^{j}$, then $[f(z)]_{n}=\sum_{j=0}^{n} f_{j} z^{j}$ ) and $\widehat{Q}_{n}(0)=Q_{n}^{*}(0)$, and therefore $\lambda=1$. These results give us the following

LEMMA 4. If

$$
\Omega_{n, 2}=s^{n} \min \left\{k_{*}^{n} \mu_{k}(n), k_{* *}^{n}\right\}
$$

then

$$
\Omega_{n, 2} \widehat{Q}_{n}\left(\frac{r}{s}\right), \quad \Omega_{n, 2} \widehat{P}_{n}\left(\frac{r}{s}\right) \in \mathbb{Z}
$$

In many cases the coefficients $a_{j}$ of the polynomial $Q_{n}(z)$ have common factors. We follow Chudnovsky's [5] ideas to cancel them out. Recently his ideas were used successfully by Rukhadze [15] and Hata $[10,11,12]$, and in [13] we gave a further approach to apply these ideas. Our main tool is the following lemma from [13] giving a criterion for primes $p>c_{6} \sqrt{n}$ dividing the numbers (5).

We use for a rational number $r$ the notation $p \mid r$ or $r \equiv 0(\bmod p)$, if $v_{p}(r) \geqslant$ 1. Further, if $v_{p}(r) \geqslant 0$, then there exists a unique $\bar{r} \in\{0,1, \ldots, p-1\}$ satisfying $\bar{r} \equiv r$ $(\bmod p)$.

LEMMA 5. (Divisibility criterion for the coefficients of $Q_{n}$ ) Let $P(l, m, n)$ denote the set of all primes satisfying $p \nmid \not F H$,

$$
\begin{gathered}
p^{2}>\max \left\{l, \max _{0 \leqslant j \leqslant l}\{|E+(n-j) F|,|G+(m-j) H|\}\right\} \\
\overline{n+B}+\overline{m+C}+1 \leqslant \bar{l} .
\end{gathered}
$$

Then

$$
\left(\prod_{p \in P(l, m, n)} p\right) \mid\left(\begin{array}{c}
n+B \\
i
\end{array}\right)\left(\begin{array}{c}
m+C \\
l-i
\end{array}\right) \quad(i=0,1, \ldots, l)
$$

Now we apply this criterion to our cases 1 and 2 .

Case 1: Let $k \in \mathbb{N}, k \geqslant 2, B=1 / k-1, C=0$. The inequality (9) has now the form $(l=n)$

$$
\overline{n+\frac{1-k}{k}}+\bar{m}+1 \leqslant \bar{n}
$$


If $\overline{n+(1-k) / k}=\bar{n}+\overline{(1-k) / k}(<p)$, then (10) cannot hold. Thus we must have

$$
\overline{n+\frac{1-k}{k}}=\bar{n}+\overline{\frac{1-k}{k}}-p \text {. }
$$

Therefore (10) is true, if the inequalities

$$
p-\frac{\overline{1-k}}{k} \leqslant \bar{n}<p, \quad 0 \leqslant \bar{m}<p-\overline{\frac{1-k}{k}}
$$

are valid.

We consider the primes $p$ satisfying $p \geqslant c_{7} \sqrt{n}, p \equiv q^{\prime}(\bmod k)$ with some $\boldsymbol{q}^{\prime} \in$ $\{1, \ldots, k-1\},\left(q^{\prime}, k\right)=1$. There then exists a unique $q \in\{1, \ldots, k-1\},(q, k)=1$, satisfying $q q^{\prime} \equiv-1(\bmod k)$. When $q^{\prime}$ runs over the reduced residue classes $(\bmod k)$, so does also $q$. Thus

$$
\overline{\frac{1-k}{k}}=\frac{1-k+q p}{k} .
$$

It follows that $p$ satisfies (10), if

$$
p\left(1-\frac{q}{k}\right)-\frac{1-k}{k} \leqslant \bar{n}<p, \quad 0 \leqslant \bar{m}<p\left(1-\frac{q}{k}\right)-\frac{1-k}{k}
$$

or

$$
\frac{n}{N+1}<p \leqslant \frac{k n+1-k}{k N+k-q}, \quad \frac{k m+1-k}{k M+k-q}<p \leqslant \frac{m}{M},
$$

where we have used the notation $\bar{n}=n-N p, \bar{m}=m-M p$. These inequalities give the following conditions and the corresponding intervals, where all the primes $p \equiv q^{\prime}$ $(\bmod k)$ satisfy $(10)$ :

(i) $k \leqslant(\beta+1) q$

$$
\begin{aligned}
& \text { (i1) } \beta N+\beta>M \geqslant \beta N+\beta-1+q / k, \quad(n /(N+1), m / M) \text {; } \\
& \text { (i2) } \beta N+\beta-1+q / k>M>\beta N+\beta(1-q / k), \\
& \quad((k m+1-k) /(k M+k-q), m / M) \\
& \text { (i3) } \beta N+\beta(1-q / k) \geqslant M>\beta N+(\beta-1)(1-q / k) \\
& \\
& \quad((k m+1-k) /(k M+k-q),(k n+1-k) /(k N+k-q))
\end{aligned}
$$

(ii) $k>(\beta+1) q$

$$
\begin{aligned}
\text { (ii1) } & \beta N+\beta>M>\beta N+\beta(1-q / k), \quad(n /(N+1), m / M) \\
\text { (ii2) } & \beta N+\beta(1-q / k) \geqslant M \geqslant \beta N+\beta-1+q / k \\
& (n /(N+1),(k n+1-k) /(k N+k-q)) \\
\text { (ii3) } & \beta N+\beta-1+q / k>M>\beta N+(\beta-1)(1-q / k) \\
& ((k m+1-k) /(k M+k-q),(k n+1-k) /(k N+k-q))
\end{aligned}
$$


After getting these intervals we use the prime number theorem in arithmetic progressions to find out an asymptotic for the part of the common factor obtained from the primes $p \equiv q^{\prime}(\bmod k)$ (for effectivity we refer to Adleman-Pomerance-Rumely [1]). Multiplying these we then get an asymptotic for the total common factor.

If we choose $\beta=x / y, x, y \in \mathbb{N},(x, y)=1$, and $N=y K+i, i \in\{0, \ldots, y-1\}$, then (i1) is of the form

$$
x K+\beta i+\beta>M \geqslant x K+\beta i+\beta-1+\frac{q}{k} .
$$

Thus the condition

$$
k \leqslant(\beta+1) q, \quad \beta i+\beta \neq[\beta i+\beta]=\left\{\begin{array}{l}
\beta i+\beta-1+q / k \text { or } \\
{[\beta i+\beta+q / k]}
\end{array}\right.
$$

gives us an integer $M=x K+[\beta i+\beta]$ satisfying (i1). The corresponding interval is

$$
\left(\frac{n}{y K+i+1}, \frac{[\beta n]}{x K+[\beta i+\beta]}\right) \text {. }
$$

For fixed $i$ and $q$ satisfying (12) we can calculate the asymptotic contribution $e^{n \Sigma_{i, q, 1}}$ for the common factor, where

$$
\begin{aligned}
\Sigma_{i, q, 1} & =\frac{1}{y \phi(k)} \sum_{K=0}^{\infty}\left(\frac{1}{K+[\beta i+\beta] / x}-\frac{1}{K+(i+1) / y}\right) \\
& =\frac{1}{y \phi(k)}\left(\Psi\left(\frac{i+1}{y}\right)-\Psi\left(\frac{[\beta i+\beta]}{x}\right)\right) .
\end{aligned}
$$

The same interval (13) and $\Sigma_{i, q, 1}$ is obtained, if the pair $(i, q)$ satisfies the condition

$$
k>(\beta+1) q, \quad \beta i+\beta \neq[\beta i+\beta]=\left[\beta i+\beta\left(1-\frac{q}{k}\right)\right]+1
$$

given by (ii1). If the pair $(i, q)$ satisfies neither the condition (12) nor (14), we set $\Sigma_{i, q, 1}=0$. The inequality (i2) is of the form

$$
x K+\beta i+\beta-1+\frac{q}{k}>M>x K+\beta i+\beta\left(1-\frac{q}{k}\right),
$$

which may give more than one integer solution $M$, if $(\beta+1) q / k>2$. More precisely, let us define

$$
I=\left[\beta i+\beta-1+\frac{q}{k}\right]-\left[\beta i+\beta\left(1-\frac{q}{k}\right)\right],
$$




$$
h= \begin{cases}0, & \text { if } \beta i+\beta-1+q / k \neq[\beta i+\beta-1+q / k], \\ 1, & \text { if } \beta i+\beta-1+q / k=[\beta i+\beta-1+q / k] .\end{cases}
$$

Thus the condition

$$
k \leqslant(\beta+1) q, \quad I-h \geqslant 1
$$

gives $I-h$ integer solutions $M={ }^{\prime} x K+[\beta i+\beta-1+q / k]-j, j=h, \ldots, I-1$, satisfying (i2). These all give disjoint intervals and we thus have the asymptotic $e^{n \Sigma_{i, q, 2}}$, where

$$
\Sigma_{i, q, 2}=\frac{1}{y \phi(k)} \sum_{j=h}^{I-1}\left(\Psi\left(\frac{[\beta i+\beta+q / k]-j-q / k}{x}\right)-\Psi\left(\frac{[\beta i+\beta-1+q / k]-j}{x}\right)\right) .
$$

The condition (ii2) is transformed to

$$
\left\{\begin{aligned}
k>(\beta+1) q, \quad\left[\beta i+\beta\left(1-\frac{q}{k}\right)\right] & =\left[\beta i+\beta-1+\frac{q}{k}\right]+1 \text { or } \\
& {\left[\beta i+\beta\left(1-\frac{q}{k}\right)\right]=\beta i+\beta\left(1-\frac{q}{k}\right) \text { or } } \\
{\left[\beta i+\beta-1+\frac{q}{k}\right] } & =\beta i+\beta-1+\frac{q}{k}
\end{aligned}\right.
$$

leading to the asymptotic $e^{n \Sigma_{i, q, 2}}$, where

$$
\Sigma_{i, q, 2}=\frac{1}{y \phi(k)}\left(\Psi\left(\frac{i+1}{y}\right)-\Psi\left(\frac{i+1-q / k}{y}\right)\right) .
$$

Again, if $(i, q)$ satisfies neither the condition (15) nor (16), let $\Sigma_{i, q, 2}=0$. The remaining cases (i3) and (ii3) give us the conditions

$$
\begin{aligned}
k \leqslant(\beta+1) q, \quad\left[\beta i+\beta\left(1-\frac{q}{k}\right)\right] & =\left[\beta i+(\beta-1)\left(1-\frac{q}{k}\right)\right]+1, \\
k>(\beta+1) q, \quad \beta i+\beta-1+\frac{q}{k} & \neq\left[\beta i+\beta-1+\frac{q}{k}\right] \\
& =\left[\beta i+(\beta-1)\left(1-\frac{q}{k}\right)\right]+1,
\end{aligned}
$$

respectively, and the asymptotic in both cases is $e^{n \Sigma_{i, q, 3}}$, where

$$
\Sigma_{i, q, 3}=\frac{1}{y \phi(k)}\left(\Psi\left(\frac{[\beta i+(\beta-1)(1-q / k)]+2-q / k}{x}\right)-\Psi\left(\frac{i+1-q / k}{y}\right)\right) .
$$

As before, we set $\Sigma_{i, q, 3}=0$, if $(i, q)$ satisfies neither the condition (17) nor (18). All the intervals obtained from the conditions (12), (14), (15), (16), (17) and (18) are disjoint. 


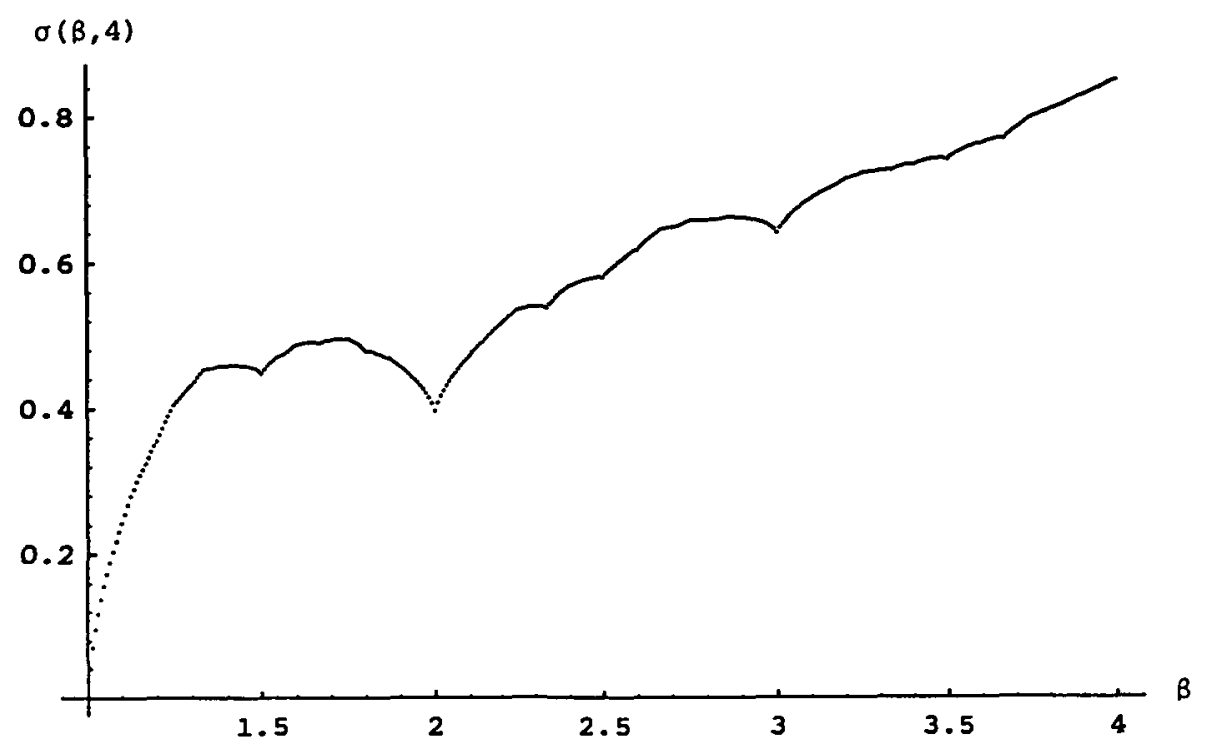

Picture 1

Thus the multiplication of the contributions of all these cases gives us the asymptotic $e^{n \sigma(\beta, k)}$ of the common factor $D_{n, 1}$, where

$$
\sigma(\beta, k)=\sum_{(i, q, t)} \Sigma_{i, q, t}
$$

the summation running over the triples $(i, q, t), q=1, \ldots, k-1,(q, k)=1, i=$ $0, \ldots, y-1, t=1,2,3$. Picture 1 shows the graph of $\sigma(\beta, 4)$ (the interval of subsequent values of $\beta$ is of length $1 / 120$ ).

Case 2 (the binomial series): Let $k \in \mathbb{N}, k \geqslant 3, B=1 / k-1, C=-1 / k$. Let further $n=n^{\prime}+1, l=m=n^{\prime}$ so that the criterion (9) has the form

$$
\overline{n+1 / k}+\overline{n-1 / k}+1 \leqslant \bar{n},
$$

where we have dropped the apostrophes.

Again we consider large primes $p$ satisfying $p \equiv q^{\prime}(\bmod k)$ for some $q^{\prime} \in$ $\{1, \ldots, k-1\},\left(q^{\prime}, k\right)=1$, and choose $q \in\{1, \ldots, k-1\},(q, k)=1$, such that $q q^{\prime} \equiv-1$ $(\bmod k)$. Then

$$
\overline{\frac{1}{k}}=\frac{q p+1}{k}, \overline{-\frac{1}{k}}=\frac{(k-q) p-1}{k} .
$$

First suppose that

$$
\bar{n}+\frac{\overline{1}}{k}<p, \quad \bar{n}+\overline{\left(-\frac{1}{k}\right)}<p .
$$


In this case $(20)$ is of the form $\bar{n}+p+1 \leqslant 0$, which is impossible. By similar reasons the cases $\bar{n}+\overline{1 / k} \geqslant p, \bar{n}+\overline{(-1 / k)}<p$ and $\bar{n}+\overline{1 / k}<p, \bar{n}+\overline{(-1 / k)} \geqslant p$ give no contribution. Thus, we are left with the case

$$
\bar{n}+\overline{\overline{1}} \geqslant p, \quad \bar{n}+\overline{\left(-\frac{1}{k}\right)} \geqslant p .
$$

These inequalities and (20) are satisfied if $(\bar{n}=n-N p)$

$$
\max \left\{\frac{(k-q) p-1}{k}, \frac{q p+1}{k}\right\} \leqslant n-N p<p
$$

or

$$
\frac{n}{N+1}<p \leqslant \min \left\{\frac{k n+1}{k N+k-q}, \frac{k n-1}{k N+q}\right\}
$$

This gives us an interval

$$
\left(\frac{n}{N+1}, \frac{k n+1}{k N+k-q}\right)
$$

if $q<k / 2$, and an interval

$$
\left(\frac{n}{N+1}, \frac{k n-1}{k N+q}\right)
$$

if $q>k / 2$.

Therefore the above intervals imply for the common factor $D_{n, 2}$ the asymptotic $e^{n \tau(k)}$, where

$$
\tau(k)=\frac{2}{\phi(k)} \sum_{\substack{k / 2<q<k \\(q, k)=1}} \sum_{N=0}^{\infty}\left(\frac{1}{N+q / k}-\frac{1}{N+1}\right)=\frac{2}{\phi(k)} \sum_{\substack{k / 2<q<k \\(q, k)=1}}\left(\Psi(1)-\Psi\left(\frac{q}{k}\right)\right)
$$

\section{ProOfS OF THE THEOREMS}

We shall use the following well-known results (see, for example, Chudnovsky [5, Corollary 3.3] and [13, Lemma 7]). Let $x>0$ and $y<0$ be given. Suppose that for each $\varepsilon>0$ there exists a constant $c_{8}$ and rational integers $p_{n}, q_{n}$ satisfying for all $n \geqslant c_{8}$ the inequalities

$$
\begin{gathered}
\frac{1}{n} \ln \max \left\{\left|q_{n}\right|,\left|p_{n}\right|\right\}<x+\varepsilon, \\
y-\varepsilon<\frac{1}{n} \ln \left|r_{n}\right|_{v}<y+\varepsilon,
\end{gathered}
$$


where $r_{n}=q_{n} \alpha-p_{n}$. Then the number $\alpha \in Q_{v}$ has an irrationality measure $m_{v}(\alpha)$ not greater than

$$
\begin{cases}1-\frac{x}{y}, & \text { if } v=\infty, \\ 1-\frac{x}{y+x}, & \text { if } v \text { is finite and } x+y<0 .\end{cases}
$$

The lower bound in (22) is not needed, if $p_{n} q_{n+1}-q_{n} p_{n+1} \neq 0$ for all $n \geqslant c_{9}$.

In Case 1 we have an approximation formula

$$
r_{n}=q_{n 2} F_{1}\left(\begin{array}{cc|c}
1, \frac{1}{k} & r \\
1+\frac{1}{k} & \frac{1}{s}
\end{array}\right)-p_{n}
$$

where

$$
q_{n}=\frac{\Omega_{n, 1} Q_{n}(r / s)}{D_{n, 1}}, \quad p_{n}=\frac{\Omega_{n, 1} P_{n}(r / s)}{D_{n, 1}}
$$

are integers. Using Lemma 1 and the asymptotic bounds for $\Omega_{n, 1}$ (see $[13$, Lemma 5]) and $D_{n, 1}$ we get the following lemma.

Lemma 6. Let $\varepsilon>0$ be given. If $|r / s|<1$ and $R(\beta, k)<1$, then

$$
\begin{gathered}
\max \left\{\left|p_{n}\right|,\left|q_{n}\right|\right\} \leqslant Q(\beta, k)^{(1+\varepsilon) n}, \\
R(\beta, k)^{(1+\varepsilon) n} \leqslant\left|r_{n}\right| \leqslant R(\beta, k)^{(1-e) n}
\end{gathered}
$$

for all $n \geqslant c_{10}$.

Therefore we immediately achieve the estimate

$$
\left.m\left({ }_{2} F_{1}\left(\begin{array}{c}
1, \frac{1}{k} \\
1+\frac{1}{k}
\end{array}\right) \frac{r}{s}\right)\right) \leqslant 1-\frac{\ln Q(\beta, k)}{\ln R(\beta, k)}
$$

for any rational $\beta \geqslant 1$ satisfying $R(\beta, k)<1$, which proves Theorem 1 .

In the corresponding $p$-adic case we choose $\beta=1, p \not k$ and $|r / s|_{p}<1$. Thus Lemma 1 and Lemma 5 give

Lemma 7. Let $\varepsilon>0$ be given and suppose $r / s>1$. Then

$$
\begin{gathered}
\max \left\{\left|p_{n}\right|,\left|q_{n}\right|\right\} \leqslant\left(\min \left\{k^{*} \mu_{k}, k^{* *}\right\} e^{\lambda(k)}|r|\right)^{(1+\varepsilon) n}, \\
\left|r_{n}\right|_{p} \leqslant|r|_{p}^{(2-\varepsilon) n}
\end{gathered}
$$

for all $n \geqslant c_{11}$.

Now the use this lemma and the condition

$$
\min \left\{k^{*} \mu_{k}, k^{* *}\right\} e^{\lambda(k)} r|r|_{p}^{2}<1,
$$


implies

$$
m_{p}\left({ }_{2} F_{1}\left(\begin{array}{c|c}
1, \frac{1}{k} & r \\
1+\frac{1}{k} & \frac{r}{s}
\end{array}\right)\right) \leqslant \frac{2 \ln |r|_{p}}{2 \ln |r|_{p}+\ln r+\lambda(k)+\ln \min \left\{k^{*} \mu_{k}, k^{* *}\right\}} .
$$

Part 2 is proved similarly using corresponding parts of Lemma 1 . This completes the proof of Theorem $1 p$.

In the second case we have

$$
r_{n}=q_{n 2} F_{1}\left(\begin{array}{c|c}
1, \frac{1}{k} & \frac{r}{s} \\
1
\end{array}\right)-p_{n}
$$

where

$$
q_{n}=\frac{\Omega_{n, 2} \widehat{Q}_{n}(r / s)}{D_{n, 2}}, \quad p_{n}=\frac{\Omega_{n, 2} \widehat{P}_{n}(r / s)}{D_{n, 2}}
$$

are integers. Further, we denote

$$
\begin{aligned}
\omega_{2} & =\min \left\{k_{*} \mu_{k}, k_{* *}\right\} e^{-\tau(k)}, \\
Q(k) & =\omega_{2}(\sqrt{s}+\sqrt{s-r})^{2}, \\
R(k) & =\omega_{2}(\sqrt{s}-\sqrt{s-r})^{2} .
\end{aligned}
$$

Analogously to Lemma 6 we thus have

Lemma 8. Let $\varepsilon>0$ be given and let $|r / s|<1, R(k)<1$. Then

$$
\begin{aligned}
& \max \left\{\left|p_{n}\right|,\left|q_{n}\right|\right\} \leqslant Q(k)^{(1+e) n}, \\
& R(k)^{(1+\varepsilon) n} \leqslant\left|r_{n}\right| \leqslant R(k)^{(1-\varepsilon) n}
\end{aligned}
$$

for all $n \geqslant c_{12}$.

Clearly we get the result

$$
m\left(\left(1-\frac{r}{s}\right)^{-1 / k}\right) \leqslant 1-\frac{\ln Q(k)}{\ln R(k)}
$$

Thus Theorem 2 is proved.

By similar considerations we achieve the cases 1 and 2 in Theorem $2 p$ using the corresponding polynomial bounds in Lemma 1. In our examples the $p$-adic values

$$
(1-z)^{1 / k}={ }_{2} F_{1}\left(\begin{array}{c}
1,-\frac{1}{k} \\
1
\end{array} \mid z\right) \quad\left(z \in \mathbb{Q}, \quad|z|_{p}<1\right)
$$


are the unique solutions of the equation

$$
x^{k}=1-z
$$

satisfying the property

$$
x \equiv 1(\bmod p)
$$

In these cases we denote

$$
(1-z)^{1 / k}=\sqrt[k]{1-z}
$$

For example

$$
\sqrt[3]{1-\frac{3 \cdot 5^{2}}{4^{3}}}=\frac{\sqrt[3]{11}}{\sqrt[3]{(-4)^{3}}}=-\frac{1}{4} \sqrt[3]{11} \in \mathbb{Q}_{5}
$$

REMARK. All the numerical computations were made using MATHEMATICA programs.

\section{REFERENCES}

[1] L.M. Adleman, C. Pomerance and R.S. Rumely, 'On distinguishing prime numbers from composite numbers', Ann. of Math. 117 (1983), 173-206.

[2] A. Baker, 'Rational approximation to $\sqrt[3]{2}$ and other algebraic numbers', Quart. J. Math. Oxford Ser. (2) 15 (1964), 375-383.

[3] P. Bundschuh P., 'Zur Approximation gewisser p-adischer algebraisher Zahlen durch rationalen Zahlen', J. Reine Angew. Math 265 (1974), 154-159.

[4] G.V. Chudnovsky, 'Number theoretic applications of polynomials with rational coefficients defined by extremality conditions', in Arithmetic and geometry (Birkhäuser, Boston, 1983), pp. 61-105.

[5] G.V. Chudnovsky, 'On the method of Thue-Siegel', Ann. of Math. 117 (1983), 325-382.

[6] L.V. Danilov, 'Rational approximations of some functions at rational points', (in Russian), Mat. Zametki 24 no. 4 (1978), 449-458.

[7] A.K. Dubitskas, 'Approximation of $\pi / \sqrt{3}$ by rational fractions', (in Russian), Vestnik Moskov. Univ. Ser. I Mat. Mekh. 42 no. 6 (1987), 73-76.

[8] D. Easton, 'Effective irrationality measures for certain algebraic numbers', Math. Comp. 46 (1986), 613-622.

[9] H.A. Erdélyi, W. Magnus, F. Oberhettinger and F.G. Tricomi, Higher transcendental functions 1 (McGraw-Hill Book Company, Inc., New York, 1953).

[10] M. Hata, 'Legendre type polynomials and irrationality measures', J. Reine Angew. Math. 407 (1990), 99-125.

[11] M. Hata, 'Irrationality measures of the values of hypergeometric functions', Acta Arith. LX.4 (1992), 335-347.

[12] M. Hata, 'Rational approximations to $\pi$ and some other numbers', Acta Arith. LXIII.4 (1993), 335-349. 
[13] A. Heimonen, T. Matala-aho and K. Väänänen, 'On irrationality measures of the values of Gauss hypergeometric function', Manuscripta Math. 81 (1993), 183-202.

[14] M. Huttner, 'Irrationalité de certaines integrales hypergéométriques', J. Number Theory 26 (1987), 166-178.

[15] E.A. Rukhadze, 'Lower estimate for rational approximations of $\ln 2$ ', Vestnik Moskov. Univ. Ser. I Mat. Mekh. 42, no. 6 (1987), 25-29.

[16] A.J. van der Poorten, 'On the arithmetic nature of definite integrals of rational functions', Proc. Amer. Math. Soc. 29 (1971), 451-456.

Department of Mathematics

University of Oulu

90570 Oulu

Finland 